\title{
Erratum: On iterated torus knots and transversal knots
}

\author{
William W Menasco
}

\begin{abstract}
In [3] the author's Theorems 1.1 and 1.2, combined, implied that iterated torus knots are transversally simple. This result is in error and this erratum pinpoints the error. In [4] a more subtle result is proven resulting in giving a geometric realization of the Honda-Etnyre transverse $(2,3)$-cable of the $(2,3)$-torus knot example (appendix joint with H Matsuda).
\end{abstract}

57M27, 57N16; 57R17, 37F20

In [3] the author's Theorems 1.1 and 1.2, combined, implied that iterated torus knots are transversally simple. The previous best result in this direction had been the proof, due to John Etnyre, that positive torus knots are transversally simple.

After the publication of [3], Etnyre and Honda argued in [2], using very different techniques from those in [3], that among the infinitely many Legendrian knots whose topological knot type is that of the $(3,2)$-cable on the $(3,2)$-torus knot, there must be one whose transversal pushoff cannot be transversally simple. This lead to a review of the proof in [3] and the discovery of an error.

To pinpoint the error, we recall that in [1] it was established that exchange reducible closed braids representing oriented knots are transversally simple when the braids are viewed as transversal knots in the standard contact structure of $\mathbb{R}^{3}$ or $S^{3}$. The author's Theorem 1.1 of [3] asserted that all iterated torus knots are exchange reducible. Theorem 1.2 then followed. The error occurs in the proof of Lemma 5.13 of [3], needed for the proof of Theorem 1.1. The changes made in the passage from Figure 15b to $16 \mathrm{~b}$ is not valid, due to an obstruction which will be described in detail in [4]. In said manuscript we will be able to recover the major part of the claimed results, and to show exactly why and how the examples pointed to in [2] and other related examples fail to be transversally simple.

Without introducing major new techniques, we can only claim a simplified version of Theorems 1.1 and 1.2 of [3]:

Theorem 1 Torus knots are exchange reducible, and hence transversally simple.

A deeper understanding of the error in Lemma 5.13 yields a complete understanding of which iterated torus knots are exchange reducible. See [4].

Acknowledgement The author is partially supported by NSF grant \#DMS 0306062. 


\section{References}

[1] J S Birman, N C Wrinkle, On transversally simple knots, J. Differential Geom. 55 (2000) 325-354 MR1847313

[2] J B Etnyre, K Honda, Cabling and transverse simplicity, Ann. of Math. (2) 162 (2005) 1305-1333 MR2179731

[3] W W Menasco, On iterated torus knots and transversal knots, Geom. Topol. 5 (2001) 651-682 MR1857523

[4] W W Menasco, An addendum on iterated torus knots arXiv:math.GT/0610566 Appendix joint with H Matsuda

Department of Mathematics, University at Buffalo

Buffalo, New York, 14260, USA

menasco@math.buffalo.edu

Received: 29 October 2006 Revised: 2 May 2007 